\title{
Zero On-Site Testing Strategies for Wireless TCMS
}

\author{
Bouaziz, Maha; Yan, Ying; Soler, Jose; Kassab, Mohamed; Berbineau, Marion
}

Published in:

IEEE Communications Magazine

Link to article, DOI:

10.1109/MCOM.001.1800994

Publication date:

2019

Document Version

Peer reviewed version

Link back to DTU Orbit

Citation (APA):

Bouaziz, M., Yan, Y., Soler, J., Kassab, M., \& Berbineau, M. (2019). Zero On-Site Testing Strategies for Wireless TCMS. IEEE Communications Magazine, 57(9), 64-69. [8847228].

https://doi.org/10.1109/MCOM.001.1800994

\section{General rights}

Copyright and moral rights for the publications made accessible in the public portal are retained by the authors and/or other copyright owners and it is a condition of accessing publications that users recognise and abide by the legal requirements associated with these rights.

- Users may download and print one copy of any publication from the public portal for the purpose of private study or research.

- You may not further distribute the material or use it for any profit-making activity or commercial gain

- You may freely distribute the URL identifying the publication in the public portal

If you believe that this document breaches copyright please contact us providing details, and we will remove access to the work immediately and investigate your claim. 


\title{
Zero on-Site Testing Strategies for Wireless TCMS
}

\author{
Maha Bouaziz, Ying Yan, José Soler, Mohamed Kassab \\ and Marion Berbineau, Member, IEEE
}

\begin{abstract}
Train Control and Monitoring System (TCMS) is a key element for smart railway operation and maintenance. In order to reduce costs and train weight, EU-funded projects are working on the development of Wireless TCMS. The data exchanges between trains and ground will be based on wireless technology such as WiFi or LTE (Long Term Evolution). First prototypes are available at European level. To facilitate the testing of the TCMS interface to ground systems it is important to develop tools able to represent railway environments without going on tracks (zero on-site testing). In this context, we present two strategies to simulate and emulate a complete wireless network (including the core network), based on Riverbed Modeler and OpenAirInterface platform able to take into account various Train-to-Ground contexts (environment, communication and railway networks load). This work considered LTE technology but can be transposed to other type of technology if representative system models are available. As a proof-of-concept of test environment we present results in terms of delay and delivery rates considering TCMS traffic and various railways scenarios such as train speed and wireless network load.
\end{abstract}

\section{INTRODUCTION}

$\mathbf{S}$ AFETY and non-safety related communications are vital for modern railway operations to ensure information exchanges between the different system stakeholders. The European Train Control System (ETCS) is used for the high speed lines in Europe and it is based on the Global System for Mobile communication for Railway (GSM-R). The control and command of urban trains rely on Communications-based train control (CBTC) system [1]. In parallel, wired train communication networks (TCN) are deployed aboard trains in rolling stocks for the automation of vehicle functions also called Train Control \& Monitoring System (TCMS).

During last years, several EU-funded projects have been working to mitigate limitations in current ETCS and CBTC systems. The goal consists in delivering an adaptive communication system for applications of any type of train control, based on a packet switching/IP technologies, allowing easy migration from existing systems and providing high throughput, security and safety to answer the future needs of systems, and resilient to radio technology evolution and to interference [1]. Embedded wired train communication networks are also concerned by this evolution towards "all wireless". Wireless TCMS (WTCMS) [2] refers now to a train communication network that consists of wireless networks [3].

Communicating parties using wireless communication network can be located in the same vehicle, in the same consist, in different consists and even in different trains. Reflecting this aspect the following four types of communication need to be distinguished: intra-Vehicle, intra-Consist, Consist to Consist (called Inter-Consist) and Train to Train. In addition, there is a fifth type of communication link: the Train-to-Ground (T2G).

In the first prototypes of WTCMS, in order to guarantee an efficient communication technology allowing a rapid transfer of data without loss, several public technologies such as WiFi or LTE are foreseen to replace wire systems related to the four types of communications but also for the $\mathrm{T} 2 \mathrm{G}$ link. All these wireless links should be evaluated in various and representative railway scenarios. Consequently, to avoid complex and expensive tests along railway lines, it is fundamental to develop flexible, configurable and programmable laboratory tools to support both the end-to-end validation and verification activities in laboratories, such an approach is also foreseen in automotive domain [4].

Zero on-site testing concept consists in developing software and hardware tools allowing validation and verification activities in laboratory to avoid complex and expensive trials on real sites. In this paper, we present two zero on-site testing strategies developed to evaluate the performance of the $\mathrm{T} 2 \mathrm{G}$ link of the WTCMS based on the use of public LTE network deployed along a high speed railway line. The evaluation versus different parameters such as speed or network load taking into account passengers services relies on pure simulation with 
a discreet event simulator [5] and emulation with OpenAirInterface platform [6].

The main contributions of this paper focus on the development of the simulation and emulation platforms as a proof of concept to evaluate specific TCMS traffic exchanges performances relayed on pure simulation through the use of Riverbed Modeler and emulation with the OpenAirInterface platform.

The remainder of this paper is organized as follows: The first section describes the WTCMS system and the different services. Then, the main characteristics of LTE system are presented. The pure simulation platform and the emulation platform principles are described with providing results. Finally, conclusions and perspectives are presented.

\section{Wireless TRAIN CONTROL \& MONITORING SYSTEM}

TCMS represents an interesting role in designing modern, flexible and intelligent railways that provides services answering the expectations in terms of safety and operational and maintenance requirements [7]. It consists in providing different services to drivers and maintenance staff, in order to remotely control and monitor trains for safety and passengers comfort. TCMS data services can be related or not to safety. The operational services are CCTV (Close Circuit TeleVision) used for surveillance, passenger information and others for customers like infotainment and access to Internet [8]. TCMS is based on the European IEC 61375 standard [9], and created for the real transmission of data related to the running state monitoring of the train operation for safety reasons. As specified in [9], the communication between TCMS on-board train and ground components is allowed through Mobile Communication Gateway (MCG) and Ground Communication Gateway (GCG).

Previously, the remote control was based on wired system, which was very costly. In addition, railways suffered from a hard fighting with the appropriate cables. Next, the high number of lines make the design of train control very complicated, the weight of trains very important and the maintenance very hard. All of these issues were the main factors to move to WTCMS, for the goal to reduce the train wires and provide a strong costs saving and weight reduction.
For the modern system, an intelligent Control and Monitoring System was created, for the goal to control braking and traction in real-time and to facilitate maintenance and reduce costs. Afterwards for the next generation, a full train control was integrated into an on-board TCMS based on a Data Transmission System (DTS). Indeed, DTS integrates a control system of traction-brake, a hard-wired logic and a Train Control Network (TCN) into a fully integrated TCMS. It provides a full train control like control of traction/brake, vehicle/train, passenger information and system/environment diagnostic.

The train control consists in controlling critical functions like traction and braking system status for the goal to facilitate and reduce maintenance costs related to the mechanical problems, as well as, noncritical functions like the door (close or open) and the light among others. In addition, it controls other functions related to the train systems such as fault detection and data log of record noteworthy, which are sent within messages to the monitor system in order to react correspondingly.

TCMS monitors control and alarm circuits, equipment status and system health among others. Then, for passenger information, TCMS collects some information such as the public address, CCTV video, video recording, voice announcement and passenger alarm with the suitable car. In addition, TCMS provides some information for passengers such as destination, delays, route, connections, platform arrival, current train location.

Therefore, by providing all these data, TCMS plays an important role for the railways safety, as it can identify faults and urgent events that damage other equipment and require regular maintenance.

TCMS outputs and inputs are used to provide an additional way for interaction between trains enddevices (ED) and ground systems for the remote control. This interaction helps providing real-time diagnostic and analyzing failure, detecting early deterioration in performance, reducing the downtime of trains and increasing the maintenance efficiency. Thus, data communications between trains and ground systems via wireless technology are required [10].

WTCMS is developed in the EU-funded CONNECTA project [10]. Fig.1 illustrates the T2G link. In the train side, collected data are gathered and sent to the ground systems via wireless connectivity 


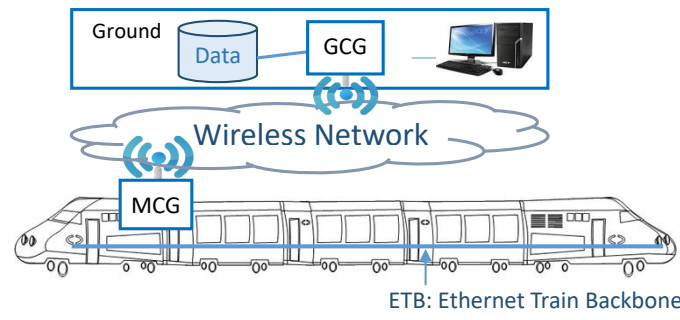

Fig. 1. TCMS Train-to-Ground link through MCG and GCG

through various MCG. In the ground side, data is received from the train through the GCG. Likewise, ground systems send data to train via wireless Communication through GCG and MCG, in order to enable an active maintenance providing high level performance. MCG and GCG applications at IP level were developed within the project and are considered for the $\mathrm{T} 2 \mathrm{G}$ validation tests.

In order to provide high level performance, some TCMS characteristics are required. First, the configuration/reconfiguration time should be in line with the time of operation of the train. For example for high intensity metro services, this time should be lower than $2 \mathrm{~min}$, for infrequent trains the targeted value is $20 \mathrm{~min}$ [7]. First, this time is used for a diagnostic test of the internal system and to identify all components in the network. Second, the response time of the system should be appropriate to allow the train driver to sense the response of the train to his actions mainly for the critical functions [7]. Third, TCMS should provide Reliability, Availability Maintainability and Safety (RAMS). All of these requirements necessitate good performance for the data exchanges between train and ground, with a high data delivery ratio and a low delay for real time applications. Thus, the key challenge for the communication Train-to-Ground of TCMS traffic consists in the choice of the suitable wireless network. This choice will depend on the availability of the network and also the availability of the frequency bands for TCMS applications. In the EU-funded Roll2rail project [2],an overview of possible applicable technologies was performed. Measurements were conducted to develop the suitable radio channel models. Finally, LTE and WiFi were chosen for performance evaluation of the wireless links. At application level, the idea is to develop an adaptable communication system as a function of wireless network availability along the railway line.

\section{LTE TECHNOLOGY FOR T2G WIRELESS LINK}

LTE technology is under deployment in Europe and also some times along railway lines by public telecommunication operators to provide connectivity to passengers. It provides packet-oriented transmission, using an all-IP approach. It also provides possible guarantees on quality of service (QoS). These advantages make transmission more flexible in managing available resources of the network, so that, it increases the network utilization.

One of the main advantage of LTE is that the LTE bearers offer class-based QoS provisioning, which consists in collecting packets with a common QoS processing. Indeed, there are two defined bearers types: Guaranteed Bit Rate (GBR) bearers for the minimum rate guarantees, and Non-GBR bearers for the best effort bearers with no resource guarantees. Each bearer is associated with different QoS Class Identifiers (QCI) characterized by the acceptable packet loss rate, priority levels and packet delay budget (A standardized QCI characteristics is defined in [11]). These bearer characteristics are very interesting in case of heterogeneous traffics, as they help classify the different traffics following their priorities to be delivered [12]. Based on these advantages, we describe in the two next sections two different strategies to test in laboratory the TCMS traffic exchanges between trains and ground systems through LTE technology that can be shared between passengers and trains.

\section{PURE SIMULATION PLATFORM FOR THE TCMS T2G LINK}

This paragraph focuses on the implementation of the pure simulation platform to evaluate performances provided by LTE for the TCMS traffic exchanges. The pure simulation is provided by Riverbed Modeler, which is based on a discreteevents simulator [5]. The use of network simulation tool to model and analyze railway train management system has been explored in several works $[12,13,14,15]$. Based on the basic functional models including Wi-Fi and LTE modules, the railway communication protocols and data services are able to be implemented and studied. Riverbed Modeler allows the modelling of the network architecture representing the test environment (TE), as well as the applications of TCMS and passengers referring to the User Equipment (UE). Moreover, it provides 


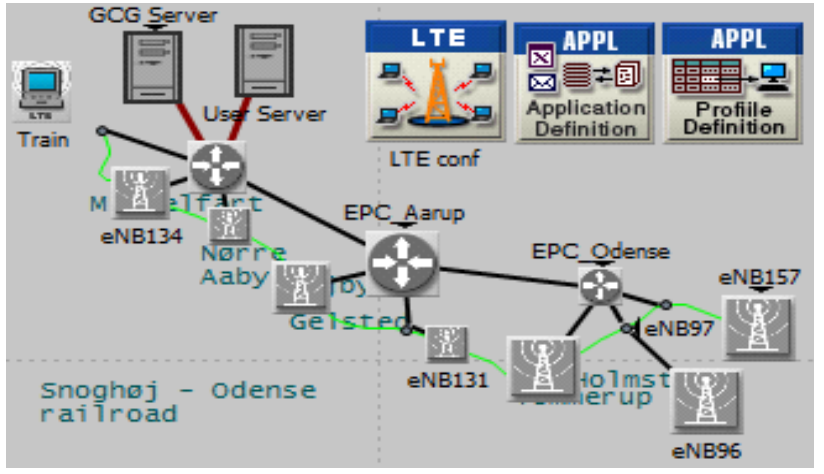

Fig. 2. Topology architecture for the Train to Ground communication

the evaluation of interaction and data exchanges in the network. With the capability of mobility feature, the movement of trains can be supported in order to examine the effect of handovers and delays, the radio channel models can be adapted to the railway environments.

As presented in Fig.2, a train is modeled as an LTE UE. ENodeBs (base stations) are deployed along the trajectory path and connected to the EPC (Evolved Packet Core) node that models the backbone functionalities and it is directly connected to the ground servers. Trains move following trajectory inspired from one of the main Danish railway line [14]. The radio and system parameters of the LTE eNodeBs are tuned taking into account the constraints of real deployment. Transmitted power, antennas gain, channel models, etc. can be changed in the Riverbed LTE module.

Simulation parameters of LTE are represented in Table I. We selected the TCMS traffics that represent the highest constraints based on Roll2Rail project [3], as follows:

- Signalling data (DL=4.6 kbps, UL=8 kbps) using TCP protocol.

- Video data (UL=1 Mbps) using UDP protocol.

- Live Voice data (UL=64 kbps) using UDP protocol.

- Recorded Voice data (UL=64 kbps) using UDP protocol.

- File data (UL=64 kbps) using TCP protocol.

TCP and UDP are two transport protocols. TCP consists in re-transmitting the lost packets when the sender did not receive an acknowledgment from the receiver during a time slot. However, UDP did not care about lost packets.

We configured three EPS bearer. The first for the
TABLE I

LTE CONFIGURATION PARAMETERS

\begin{tabular}{|c||c||}
\hline Frequency band & $920 \mathrm{MHz}(\mathrm{BW}: 5 \mathrm{MHz})$ \\
\hline eNB Transmission power & $36 \mathrm{dBm}$ \\
\hline eNB antenna height & 50 meters \\
\hline eNB antenna gain & $15 \mathrm{dBi}$ \\
\hline UE antenna gain & $1 \mathrm{dBi}$ \\
\hline Pathloss model & UMa \\
\hline Multipath channel model & ITU Vehicular A \\
\hline
\end{tabular}

signaling application with Class Identifier 3 (GBR) and with the highest priority, it guarantees $16 \mathrm{kbps}$ for the up and down traffic. The second for the Voice data with Class Identifier 2 (GBR) and with priority 5, it guarantees $64 \mathrm{kbps}$ for the up and down traffic. The third one is configured for the rest of applications without any guarantee. The Class Identifier is (N-GBR) and the priority is 9. For railways, the TCMS signaling data has the highest priority, because it represents the critical control data. We have considered here the same standard channel model for all eNodeBs taking into account mobility. The channel models can be modified to set up a more realistic test environment. For the simulations we consider two factors that may affect performance of the TCMS traffic exchange: train speed and LTE network load.

\section{Influence of train speed}

The train is moving with a high speed and exchanges data with ground devices (GCG) through the LTE network. Thus, it is necessary to keep the train connectivity during its movement, so that it can continuously provide data exchange between MCG and GCG devices. This test can show the capacity of LTE with the given configuration to transfer TCMS traffic when the train moves with a high speed $(300 \mathrm{~km} / \mathrm{h})$ using macro cells. If key performance indicators are stated by train operators for the different TCMS traffic, the system will allow the validation of the indicators varying the test environment.

\section{Influence of Network load}

For passengers comfort, LTE network can be shared between train and passengers. So that, the available bandwidth should be shared between them. The study shows the efficiency of LTE with the chosen bearer in a given congestion case. In our 
TABLE II

SIMULATION RESULTS OF TCMS TRAFFIC TRANSFER

$\left.\begin{array}{|c||c||c|}\hline & \text { PDR } & \text { delay } \\ \hline \text { Signaling data } & 100 \% & {[0.011 \mathrm{~s} . .0 .012 \mathrm{~s}}\end{array}\right]$

scenario, we consider different kind of users (representing the passengers) such as web browser, email and file transfer data; mobile user using Instant message and game data; and multimedia user using VoIP and video conference data. The developed platform is able to evaluate the performances of TCMS traffic exchanges between MCG and CGC in different congestion configurations.

After the simulation test of a congested Network, the different results prove that LTE provides appropriate performance for the transfer of TCMS data. As presented in Table II, the signaling and file data are successful transferred between MCG and GCG. However, some data are lost for the voice and video data. These results are explained by the fact that signaling and file data are sent using TCP protocol that consists in re-sending lost data, as well as, priority parameters provide by the Guaranteed Bearer Rate (GBR). Regarding video and voice data, they use UDP protocol where there is not retransmission of lost data. The video data is the worst PDR due to the fact that it uses UDP without GBR. Indeed, in a congested cell, GBR radio bearers with low priority are preempted if this is necessary and sufficient to accept a radio bearer requested for an EPS bearer with a high priority. Simulation results for a high-speed scenario along a realistic railway line shows that LTE allows the successful transfer of the interesting and critical application traffic, when passengers use LTE simultaneously.

\section{OPENAIRINTERFACE PLATFORM TO EMULATE LTE FOR TCMS PERFORMANCE EVALUATION}

This part presents the emulation platform developed considering both OpenAirInterface (OAI) and Riverbed modeler. This platform is complementary to the pure simulation one. OpenAirInterface is a platform providing an open-source software and hardware used for emulation with a high level of realism. OAI is a solution based on the Software Defined Radio (SDR) and it provides a complete implementation of the elements of the LTE (4G) system architecture. It includes all protocol stacks from physical to networking layers with standard compliant implementations (Release 8 and Release 10) of Physical, Medium-access Control, Radio Link Control, Packet Data Convergence Protocol and Radio Resource Control, for both UE and eNodeB and in both Time Division Duplex (TDD) and Frequency Division Duplex (FDD). Regarding the core network, the standard compliant implementations (Release 9 and Release 10) of a subset of 3GPP LTE EPC components are supported by OAI. OAI platform aims to perform more reliable and realistic simulations by using a real stack. Concerning the configurations of an experimentation of inlab radio network, the software platform of OAI is installed on a host computer running the full protocol stack in the emulation mode. The eNodeB experimental consists of two elements: a computer as the baseband unit, and RF hardware as the radio part. The functionality of the transceiver is realized using SDR card connected to a host computer. The OAI software is based on the $\mathrm{C}$ language and run on a low latency Linux kernel. Actually, OAI is in evolution towards the $5 \mathrm{G}$ generation. This platform allows us emulating exchanges of TCMS traffic between train and ground devices.

Figure 3 represents the proposed platform consisting of five elements: UE (representing MCG in the train side), eNodeB, EPC, OPNET (Riverbed) representing a simulated backhaul network and GCG. Connection of Riverbed to others is created thanks to the SITL (System In The Loop) tool,which allows interfacing real equipment to the network simulations by converting real to simulated traffic and vice versa. GCG application is used to transfer real TCMS traffic and to collect some information about the train. The packet transmission delays in the links between eNodeB-EPC and EPC-Simulated backhaul are set to $0.01 \mathrm{~s}$ and $0.1 \mathrm{~s}$ respectively, as well as, to $0.05 \mathrm{~s}$ in the simulated backhaul.

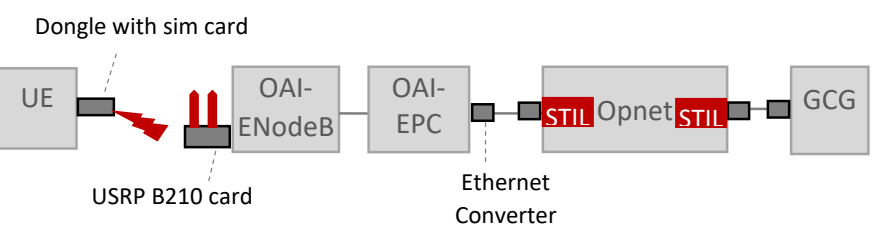

Fig. 3. Railway Platform based on OAI 


\begin{tabular}{|c|c|c|c|c|c|c|c|c|}
\hline I & Lasicomm & senvicest: & Loeation & $\begin{array}{c}\text { Truin Info } \\
\end{array}$ & $\begin{array}{l}\substack{\text { Remove } \\
\text { MOQG }} \\
\end{array}$ & $\begin{array}{c}\text { Sond Trinfo } \\
\text { roq. }\end{array}$ & $\begin{array}{c}\text { Sond LOC } \\
\text { req. }\end{array}$ & $\begin{array}{c}\text { Dotala } \\
\text { lato }\end{array}$ \\
\hline 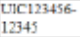 & 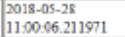 & 0 & 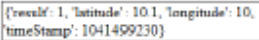 & 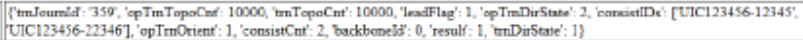 & Rempre & Tinto rea & LOCina & $\lim _{n \rightarrow \infty}$ \\
\hline
\end{tabular}

Fig. 4. Illustration of the UE connection with GCG host with location and command train information

\section{Tests and evaluation with OpenAirInterface}

After testing the successful connection between UE and GCG devices, GCG can have location and information of the Train as illustrated in Fig.4. The goal of this platform consists in verifying the possibility of connecting physical emulation tools with simulation software tools in an end-to-end communication testbed. In addition, this platform provides the emulation of the TCMS traffic exchange between UE and GCG. Emulation result shows that the needed delay to transfer packet between UE and GCG is about an average of $0.158 \mathrm{~s}$, it is between $0.141 \mathrm{~s}$ and $0.173 \mathrm{~s}$ without any loss of data. This difference in time is acceptable to have a stable transfer of TCMS data, and fulfill the requirement to the data treatment for train control. In addition, for testing purpose, we propose to add a $5 \%$ of loss in the related link between EPC and the simulated backhaul, the emulation result shows that only $3 \%$ of TCMS traffic are lost, thanks to the priority accorded to this kind of traffic. Finaly, the experimental results presented here reveals primary testing and explores the potential usage of this inlab testbed for TCMS performance evaluation.

\section{Redundancy test in backhaul network}

The purpose of this test is to examine the network simulation ability in the Riverbed Modeler. The performance measurement focuses on network availability and redundancy. We extend the simulated network backhaul from using one switch to eight switches. After the emulation test, we notice that the routing path is usually updated when some link are failed and broken.

Compared to the first configuration test, an extra link transmission delay is added on each link as by $0.01 \mathrm{~s}$. Therefore, the total transmission delay from EPC to the GCG is about $0.149 \mathrm{~s}$ compared to $0.115 \mathrm{~s}$ for the first configuration network backhaul, due to the routing packets via a longer path.
Emulation tests and evaluation results lead to the following conclusions: 1) the GCGSim simulator environment works successfully with the emulation test environment. All functions are tested and results are verified, 2) the network performance such as delay and packet loss, are examined with the GCG host services. Artificial delay and packet loss are inserted in both hardware and software environment to mimic the real network transmission environment.

\section{CONCLUSION}

Two complementary strategies have been presented in this paper, aiming to simulate and emulate TCMS traffic exchanges between GCG and MCG through LTE for a zero on site testing approach. The challenge of this work has consisted in providing flexible solutions for realistic simulation and emulation of wireless railway environment to evaluate the performances of the T2G exchanges of TCMS traffic in laboratory, with the most realistic representation of the wireless system and the T2G environment. The two strategies proposed are complementary and work correctly. As an example, in the simulation strategy, the radio channel environment is modelled using a standardized channel model. This model can be changed by more appropriate railway radio channel models while the train is moving along the network. In the emulation strategy, we showed that we are able to mimic a real wireless transmission, taking into account the whole network. The radio channel is the "real wireless link" in the laboratory. In the future, this link can be replaced by a channel emulator. This work constitutes a proof-of-concept to allow real railway equipment testing without the need for infield tests on tracks (zero on-site testing), contributing to decrease time to market of railway systems. 


\section{ACKNOWLEDGMENT}

This work has been performed within Safe4rail project and funded from $\mathrm{H} 2020$ under grant agreement No 730830.

\section{REFERENCES}

[1] J. Moreno et al., "A survey on future railway radio communications services: challenges and opportunities," IEEE Cотmun. Mag., vol. 53, no. 10, Oct. 2015, pp. 62-68.

[2] J. Moreno et al., "The wireless train communication network: Roll2rail vision," IEEE Vehic. Tech. Mag., vol. 13, no. 3, Sep. 2018, pp. 135-143.

[3] Roll2rail-2017, "D2.1: Specification of the Wireless TCMS," 2017.

[4] H. Fouchal et al., "A validation tool for cooperative intelligent transport systems," JCS, vol. 22, no. 2, Jun. 2017, pp. 283-288.

[5] Riverbed modeler, 2017; http://www.riverbed.com

[6] 5g software alliance for democratising wireless innovation; http://www.openairinterface.org/

[7] G. Neil, "On board train control and monitoring systems," IET 13th Professional Development Course on Electric Traction Systems 2014, London, UK., 2014.

[8] C. Liu, X. Li, and P. Yang, "Train control management systemsafety assessment," Springer EITRT2013. Lecture Notes in Electrical Engineering, Berlin, Heidelberg, 2013

[9] IEC Standard, "IEC 61375-2-6: Electronic Railway equipment train communication network (TCN): Part 2-6: On-board to Ground Communication," 2016.

[10] CONNECTA-2017, "D2.1: Requirements and Specification for the T2G System. Contributing to Shift2Rail's next generation of high capable and safe TCMS and brakes," 2017.

[11] S. Sesia, I. Toufik, and M. Baker, "LTE - The UMTS Long Term Evolution: From Theory to Practice," John Wiley Sons, Ltd, 2009.

[12] A. Khayat et al., "Lte based communication system for urban guided-transport: A qos performance study," Springer, Nets4cars,Nets4Trains, Berlin, Heidelberg, 2013, pp. 197-210.

[13] C. Pinedo et al., "Modelling and simulation of ERTMS for current and future mobile technologies," IJVT, vol. 9, no. 2, 2015.

[14] A. Sniady and J. Soler, "Lte for railways: Impact on performance of etcs railway signaling," IEEE Vehic. Tech. Mag., vol. 9, no. 2, Jun. 2014, pp. 69-77.

[15] J. Farooq et al., "A multiradio, multi-hop ad-hoc radio communication network for communications-based train control (cbtc): Introducing frequency separation for train-to-track side communication," 15th IEEE Annual CCNC, Las Vegas, USA, Jan. 2018, pp. 1-7.

Maha Bouaziz is currently occupying a Postdoctoral position at the French Institute IFSTTAR, France. She received her Ph.D. degree in computer science in 2017 from the National School of Computer
Sciences (ENSI) Tunisia and the University of Marne-la-Vallee, Paris, France. She received her Engineering Diploma in computer science from the National School of Engineers of Sfax (ENIS) in Tunisia. Her current research focuses on wireless networking, mobility management for WSNs based on the 6LoWPAN technology, and intelligent transportation systems especially on railways domain.

Ying Yan received the B.Eng. degree in electrical engineering from the Beijing University of Technology, China, in 2002. She has received her M.S. degree in electronics engineering in 2004, and the $\mathrm{Ph} . \mathrm{D}$. degree in telecommunication engineering in 2010 from Technical University of Denmark. During 2006-2007, she worked as a research scientist at the department of communication platforms in the Technical Research Centre of Finland (VTT), Finland. Ying Yan has worked in the fields of railway communication system, hybrid optical wireless network, and heterogeneous wireless networks. Her research focus is the network performance evaluation and analysis based on network simulation and emulation.

José Soler Associate Professor. Born in 1974. Received his MSc degree on Telecommunication Engineering from Zaragoza University (Spain) in 1999 and PhD degree in Electrical Engineering from DTU (Denmark) in 2005. He holds also an MBA from UNED (2016) and a degree in Management from Erhversakademiet Copenhagen Business (2010). Previous employee of ITA (Spain), ETRI (South Korea), COM DTU (Denmark) and GoIP International (Denmark). His research interests include integration of heterogeneous telecommunication networks and telecommunication software and services.

Mohamed Kassab is an assistant professor at the University of Monastir, Tunisia. He received his $\mathrm{PhD}$ in computer sciences from jointly Telecom Bretagne, France and ENSI, Tunisia in 2008. He also received the Engineer degree and the M.S. in computer sciences from ENSI, Tunisia respectively in 2003 and 2004. He is broadly interested in problems related to mobility management and link layer optimization of wireless technologies. His works are mainly related to wireless technologies use for Internet of Things and Intelligent Transport Systems.

Marion Berbineau received the Engineer degree from Polytech'Lille (France) and the Ph.D. degree from the Univ. of Lille, both in electrical engineering, respectively in 1986 and 1989. She is Research Director at IFSTTAR (Institute for Science and Technology of Transport, Development and Networks), France. She is expert in the fields of radio wave propagation in transport environments, channel characterization and modeling, wireless systems for railways. She coordinates Railway research at IFSTTAR. 